\title{
Parámetros estadísticos de las acciones permanentes en la edificación
}

\section{Statistical parameters of the permanent actions in building}

\author{
E. González-Valle ${ }^{(*)}$
}

\section{RESUMEN}

Los Códigos de acciones que especifican las acciones de peso propio y cargas permanentes actuantes sobre los forjados de edificación dan valores que tienen un carácter de acciones medias y como tal se adoptan en el proyecto de estructuras. Pero para poder realizar análisis de fiabilidad estructural o análisis probabilistas de la seguridad resulta necesario disponer además de valores del coeficiente de variación o de la desviación estándar. En el presente artículo se sugiere una pauta para determinar estos parámetros y se proponen unos valores para las soluciones empleados más usualmente de forjados unidireccionales, solados y tabiquerías en edificios de viviendas.

Palabras clave: edificación; acciones; probabilismo; parámetros estadísticos; fiabilidad estructural.

\section{ABSTRACT}

The Codes of actions that specify the actions of own weight and permanent loads acting on the building structural floors give values that have a character of average actions and as such are adopted in the project of structures. However, in order to perform structural reliability analysis or probabilistic safety analysis, it is also necessary to know the coefficient of variation or the standard deviation values. In the present article a guideline is suggested to determine these parameters and values are proposed for the most commonly used solutions of unidirectional floor structural system, floorings and partitions used in buildings.

Keywords: building; actions; probabilism; statistical parameters; structural reliability.

(*) Dr. Ing. de Caminos, Canales y Puertos.

Persona de contacto/Corresponding author: egonzalezvalle@yahoo.es (E. González-Valle).

ORCID: https://orcid.org/oooo-0002-2759-5097 (E. González-Valle).

Cómo citar este artículo/Citation: E. González-Valle (2019). Parámetros estadísticos de las acciones permanentes en la edificación. Informes de la Construcción, 71(553): e278. https://doi.org/10.3989/ic.67500

Copyright: (C) 2019 CSIC. Este es un artículo de acceso abierto distribuido bajo los términos de la licencia de uso y distribución Creative Commons Reconocimiento 4.0 Internacional (CC BY 4.0). 


\section{INTRODUCCIÓN}

Las acciones actuantes sobre una estructura son, en general, las acciones directas, (peso propio, una o más cargas permanentes, sobrecargas de utilización y climático-ambientales de nieve y viento) y las indirectas, (temperatura, reológicas debidas al comportamiento de los materiales como la fluencia, la relajación, la retracción y las deformaciones impuestas). Los valores y el carácter de estas acciones son normalmente especificados en los códigos de acciones para ser aplicadas al proyecto de estructuras de acuerdo con métodos semiprobabilistas.

Para realizar un análisis de secciones por métodos probabilistas o para realizar análisis de fiabilidad estas acciones deberían ser consideradas como variables probabilistas, según se señala en los códigos de acciones, por lo que se precisaría conocer los parámetros estadísticos que las caracterizan como variables aleatorias. En contrapartida los códigos actuales que especifican las acciones para el proyecto de estructuras de edificación establecen valores que tienen un carácter mas bien determinista.

Pretendemos sugerir en este breve articulo una pauta para calcular los parámetros estadísticos media, desviación estándar y coeficiente de variación $(\mu, \sigma$ y $\delta$ ) que caracterizan a las acciones de carácter permanente, (peso propio, solado mas revestimiento de techos y tabiquería) contempladas como variables aleatorias, para poder componerlas en un segundo paso y calcular los parámetros correspondientes a la acción total, ver Figura 1.

\section{PESO PROPIO}

Se trata de una variable probabilista que puede ser obtenida mediante el producto de otras dos variables del mismo tipo: el peso específico y el volumen, esta última a su vez compuesta y calculable como una combinación de un conjunto de dimensiones que serian asimismo variables probabilistas.
Tanto los códigos probabilistas como los actuales que contemplan procesos de dimensionamiento o comprobación de estructuras por teorías semiprobabilistas, señalan que los valores representativos de las acciones de peso propio, correspondientes a los pesos de elementos estructurales, son sus valores medios, es decir los deducidos de los volúmenes calculados con sus dimensiones nominales, y los pesos específicos medios de los materiales empleados. Asimismo, las dimensiones representativas o nominales que se especifican en proyecto son sus valores medios.

Con los criterios expuestos y adoptando para las dimensiones de los elementos estructurales que normalmente se especifican en los planos como valores representativos o nominales sus valores medios y con los pesos específicos asimismo especificados por su valores medios, es inmediato el cálculo del valor medio de las acciones de peso propio aplicando a estos volúmenes medios los pesos específicos medios del hormigón estructural que sugieren los códigos de acciones o las propuestas de códigos probabilistas.

Conocidos pues los valores medios de las acciones de peso propio de la estructura, para tener caracterizada la variable precisamos conocer la desviación estándar, con lo cual también tendríamos conocido el coeficiente de variación, y el tipo de distribución al que se ajusta.

La distribución que generalmente se sugiere aplicar a los valores de los pesos propios de los elementos estructurales es la Distribución Normal y es la que adoptaremos.

En lo referente a las desviaciones estándar de estas acciones, si se tratase de una situación de dimensionado o comprobación en fase de proyecto se procedería de manera diferente a si lo que abordásemos fuese una situación de comprobación de una estructura existente. En el primer caso los códigos deberían dar, como datos de proyecto, no solo el valor característico y representativo de las variables pesos específicos nominales y dimensiones y su carácter, es decir si se trata de valores medios o de otro tipo, sino también los valores de las

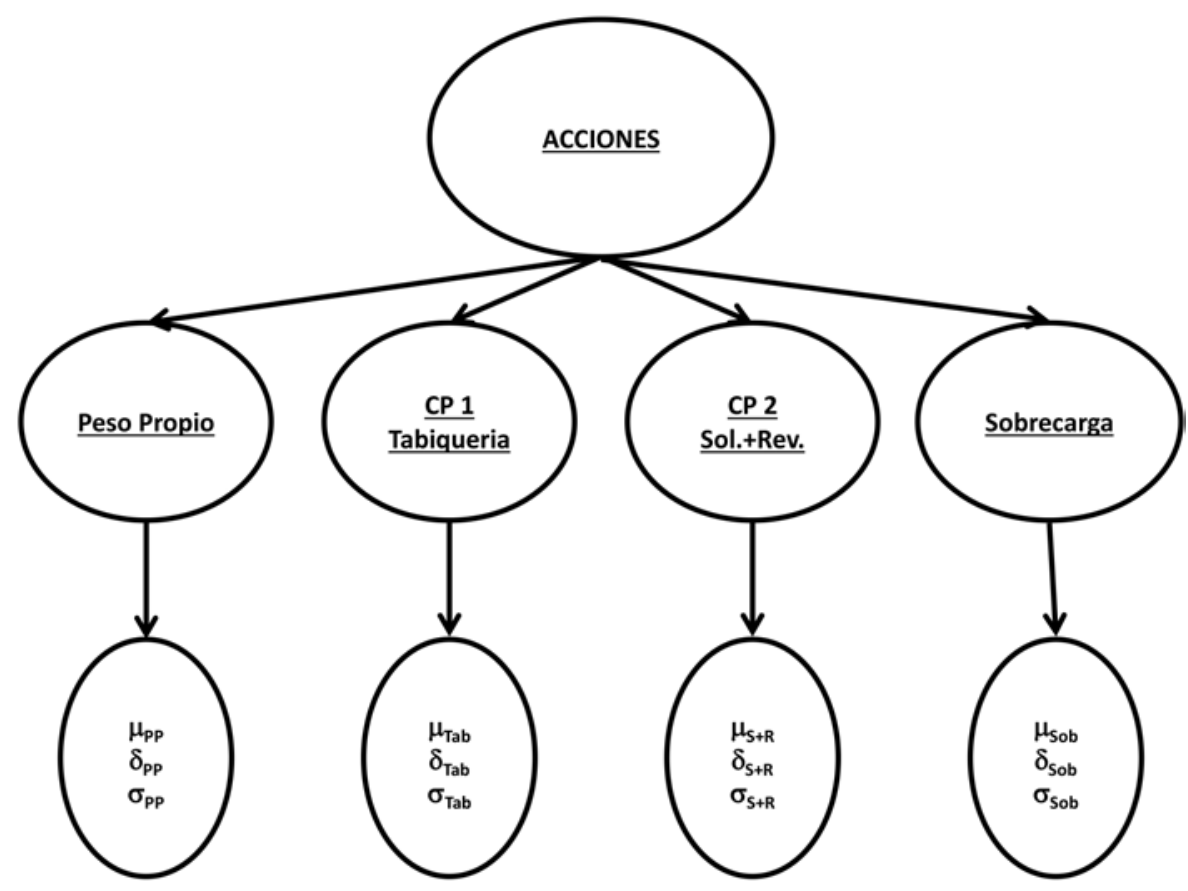

Figura 1. Parámetros estadísticos de las acciones. 
desviaciones estándar de las dimensiones y de los pesos específicos de los materiales con los que calcular la desviación estándar del peso propio como variable probabilista. En el segundo caso, comprobación de una estructura existente, los valores de las dimensiones medias y de los pesos específicos medios podrían deducirse de los datos tomados en inspecciones y ensayos realizados sobre una muestra significativa tomada de la estructura existente.

Centrándonos de momento en el caso primero, caso de proyecto o de comprobación de la estructura en caso de proyecto, y como resumen de lo expuesto, necesitamos:

- Conocer las dimensiones nominales para las que se adoptan las dimensiones medias. Estos valores son datos del proyecto y con estas dimensiones calcularemos los volúmenes medios $\left(\mathbf{V}_{\mathbf{M}}\right)$.

- Conocer las desviaciones estándar y coeficientes de variación de las dimensiones, datos que deberían figurar en los códigos estructurales. Conocidas las desviaciones estándar y los coeficientes de variación de las dimensiones, como combinación de variables podríamos conocer las desviaciones estándar $\left(\boldsymbol{\sigma}_{\mathrm{v}}\right)$ de los volúmenes y sus coeficientes de variación $\left(\boldsymbol{\delta}_{\mathbf{v}}\right)$.

- Conocer el peso específico medio $\left(\mathbf{P}_{\mathbf{e s p}, \mathbf{M}}\right)$ del material de la estructura, su desviación estándar $\left(\boldsymbol{\sigma}_{\mathrm{Pesp}}\right)$ y su coeficiente de variación $\left(\boldsymbol{\delta}_{\text {Pesp }}\right)$, datos que asimismo deberían estar especificados en los códigos estructurales.

Conocidos los datos anteriores es posible conocer los valores medios $\left(\mathbf{p p}_{\mathbf{M}}\right)$ de los pesos de los elementos estructurales, así como su desviación estándar $\left(\boldsymbol{\sigma}_{\mathrm{PP}}\right)$ y su coeficiente de variación $\left(\boldsymbol{\delta}_{\mathrm{PP}}\right)$ como variable estadística producto de las variables volumen y peso específico del material (ecuaciones [1], [2] y [3]):

$$
\mathbf{p p}_{\mathrm{M}}=\mathbf{V}_{\mathrm{M}} \cdot \mathbf{P}_{\mathrm{esp}, \mathrm{M}}
$$

$$
\begin{gathered}
\delta_{\mathrm{pp}}=\left(\delta_{\mathrm{v}}^{2}+\delta_{\mathrm{Pesp}}^{2}\right)^{0.5} \\
\sigma_{\mathrm{pp}}=\delta_{\mathrm{pp}} \cdot \mathrm{pp}_{\mathrm{M}}
\end{gathered}
$$

Como ejemplo, para el caso de un forjado de edificación, hemos desarrollado un análisis paramétrico de un forjado tipo formado por nervios de hormigón in situ con losa superior y aligeramientos cerámicos, tipología de forjado bastante usual en las edificaciones actuales, ver Figura 2.

Sobre este forjado hemos considerado variabilidades de sus dimensiones nominales $\left(\mathrm{h}, \mathrm{h}_{\mathrm{o}} \mathrm{y} \mathrm{b}\right)$ de acuerdo con la Tabla 1, contemplando un total de 125 casos para cada conjunto de dimensiones nominales.

Tabla 1. Variabilidad de las dimensiones nominales $h, h_{o} y b$

\begin{tabular}{|c|c|c|}
\hline $\begin{array}{c}\mathbf{h}_{\mathbf{o}} \\
\mathbf{c m}\end{array}$ & $\begin{array}{c}\mathbf{h} \\
\mathbf{c m}\end{array}$ & $\begin{array}{c}\mathbf{b} \\
\mathbf{c m}\end{array}$ \\
\hline $\mathrm{h}_{\mathrm{o}}-1, \mathrm{o}$ & $\mathrm{h}-2, \mathrm{o}$ & $\mathrm{b}-2, \mathrm{o}$ \\
\hline $\mathrm{h}_{\mathrm{o}}-\mathrm{o}, 5$ & $\mathrm{~h}-1, \mathrm{O}$ & $\mathrm{b}-1, \mathrm{O}$ \\
\hline $\mathrm{h}_{\mathrm{o}}=5 \mathrm{~cm}$ & $\mathrm{~h}=$ variable & $\mathrm{b}=70 \mathrm{~cm}$ \\
\hline $\mathrm{h}_{\mathrm{o}}+\mathrm{O}, 5$ & $\mathrm{~h}+1, \mathrm{O}$ & $\mathrm{b}+1, \mathrm{O}$ \\
\hline $\mathrm{h}_{\mathrm{o}}+1, \mathrm{o}$ & $\mathrm{h}+2, \mathrm{O}$ & $\mathrm{b}+2, \mathrm{O}$ \\
\hline 5 casos & 5 casos & 5 casos \\
\hline & Total 125 casos \\
\hline
\end{tabular}

A su vez hemos considerado unos cantos de forjado variables entre 15 y $35 \mathrm{~cm}(15,20,25,30$ y $35 \mathrm{~cm})$. El ancho de nervio y las restantes dimensiones, así como los pesos y dimensiones de los aligeramientos cerámicos los hemos considerado variables deterministas, dada la escasa influencia del ancho de nervio en el peso del forjado y la escasa variabilidad de la geometría y el peso del aligeramiento. En cuanto a la separa-

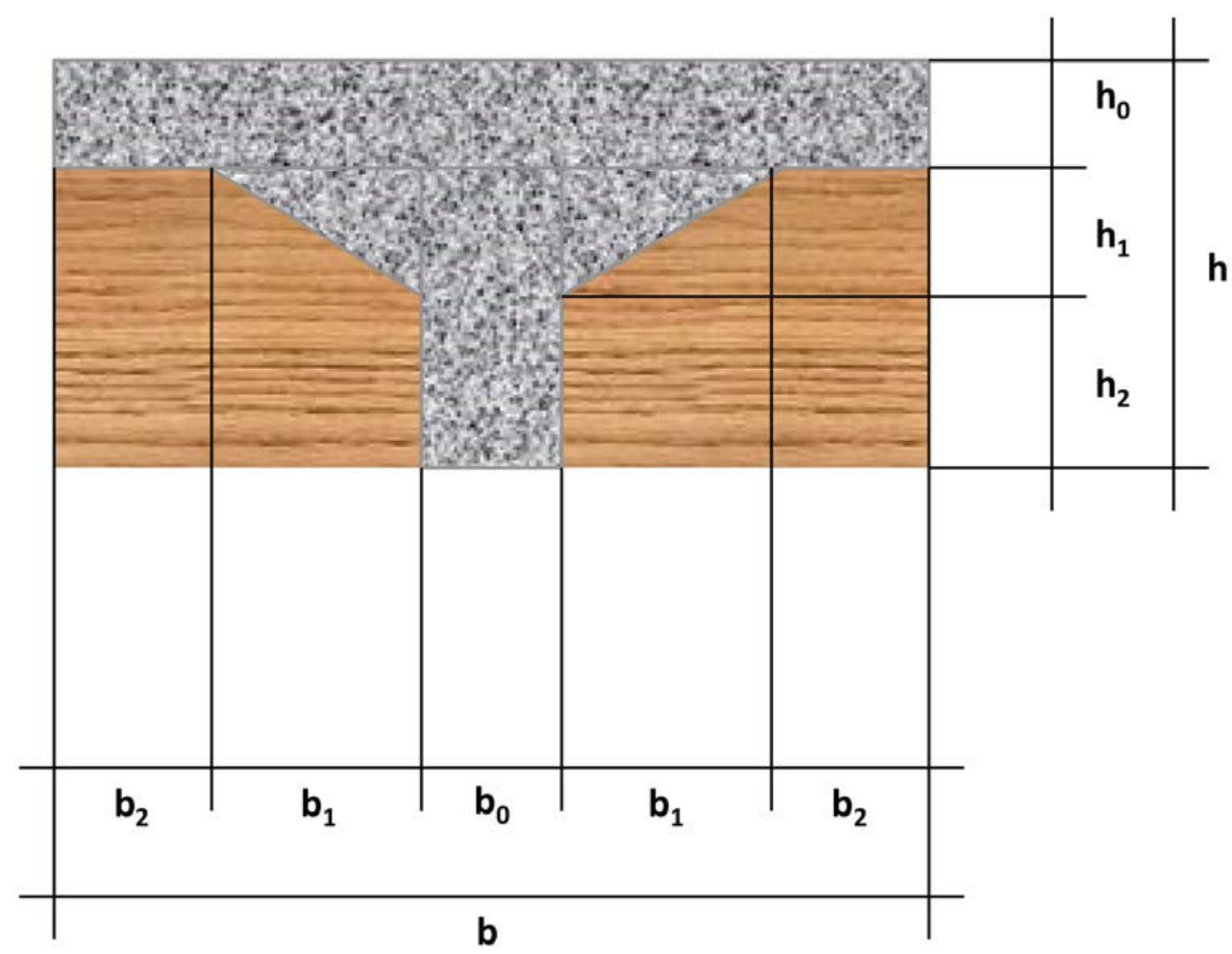

Figura 2. Forjado de ejemplo. 
ción entre nervios para todos los cantos hemos considerado un intereje nominal del forjado de $70 \mathrm{~cm}$. Según este estudio paramétrico hemos determinado los volúmenes de hormigón por metro cuadrado de forjado. Adoptando un peso específico medio $\mathbf{P}_{\text {esp, } \mathbf{M}}=24 \mathrm{kN} / \mathrm{m}^{3}$ y un coeficiente de variación del mismo de $\delta_{\text {Pesp }}=\mathbf{0 , 0 4}$ para el hormigón, según sugieren publicaciones especializadas, hemos calculado los pesos medios de cada canto de forjado $\left(\mathbf{p p}_{\mathrm{F}}\right)$, sus desviaciones estándar $\left(\boldsymbol{\sigma}_{\mathrm{F}}\right)$ y sus coeficientes de variación $\left(\boldsymbol{\delta}_{\mathrm{F}}\right)$. Asimismo hemos comprobado el buen ajuste de la variable peso del forjado a una distribución Normal.

El resumen de los valores de los pesos medios y sus coeficientes de variación se acompaña en las Tablas 2 y 3.

Tabla 2. Pesos medios

\begin{tabular}{|c|c|c|}
\hline $\begin{array}{c}\text { CANTO } \\
\mathbf{c m}\end{array}$ & $\begin{array}{c}\mathbf{\mathbf { p p }} \\
\mathbf{k N} / \mathbf{m}^{\mathbf{2}}\end{array}$ & $\begin{array}{c}\mathbf{p p}_{\mathbf{M}, \text { Ajustado }} \\
\mathbf{k N} / \mathbf{m}^{\mathbf{2}}\end{array}$ \\
\hline 15 & 2,37 & 2,40 \\
\hline 20 & 2,64 & 2,70 \\
\hline 25 & 2,91 & 3,00 \\
\hline 30 & 3,30 & 3,30 \\
\hline 35 & 3,63 & 3,60 \\
\hline
\end{tabular}

Tabla 3. Coeficientes de variación

\begin{tabular}{|c|c|c|}
\hline $\begin{array}{c}\text { CANTO } \\
\mathbf{c m}\end{array}$ & $\begin{array}{c}\text { CoV } \\
-\end{array}$ & $\begin{array}{c}\text { CoV Ajustado } \\
-\end{array}$ \\
\hline 15 & 0,078 & 0,077 \\
\hline 20 & 0,073 & 0,072 \\
\hline 25 & 0,068 & 0,068 \\
\hline 30 & 0,063 & 0,063 \\
\hline 35 & 0,060 & 0,059 \\
\hline
\end{tabular}

De acuerdo con los valores obtenidos para este tipo de forjado hemos aproximado, tanto para los pesos medios como para los coeficientes de variación, una recta sensiblemente correspondiente a un ajuste lineal, tal y como acompañamos en las Figuras 3 y 4.

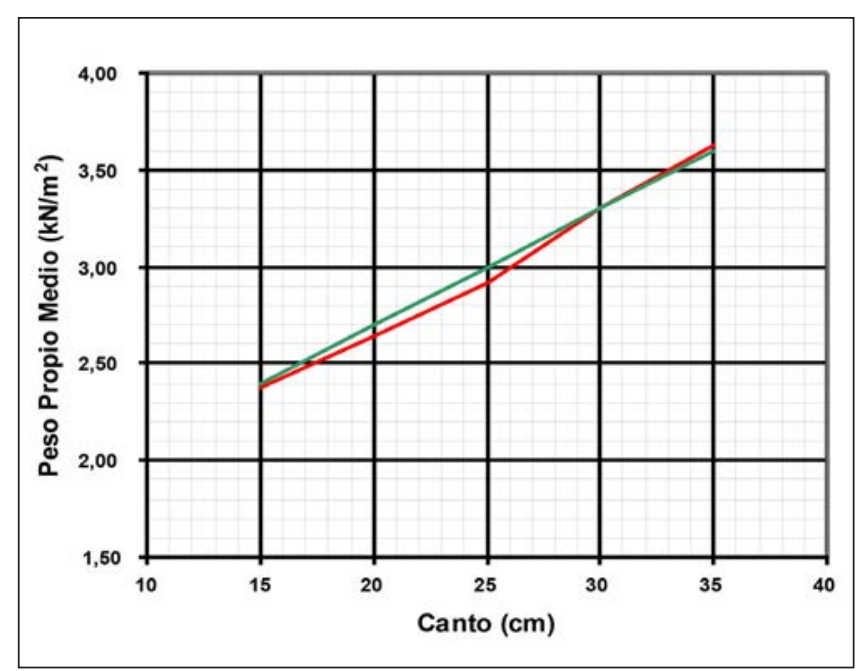

Figura 3. Aproximación para pesos medios.

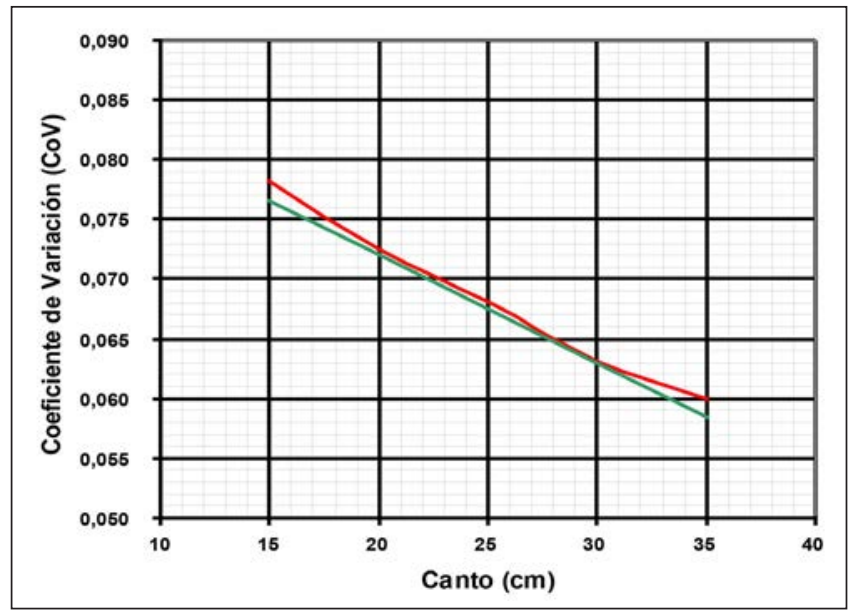

Figura 4. Aproximación para coeficientes de variación.

Con el análisis paramétrico y los ajustes realizados hemos estimado los parámetros estadísticos de la variable probabilista peso propio del forjado (ecuaciones [4], [5] y [6]):

$$
\begin{gathered}
\mathrm{pp}_{\mathrm{M}}=1,40+\mathbf{0 , 0 6} \cdot \mathrm{h} \\
\delta_{\mathrm{pp}}=0.9 \cdot(0,1-0,001 \cdot h) \\
\sigma_{p p}=\mathrm{pp}_{\mathrm{M}} \cdot \delta_{\mathrm{pp}}
\end{gathered}
$$

para $\mathbf{h}$, canto del forjado, en $\mathbf{c m} \mathbf{y} \mathbf{p p}_{\mathbf{M}}$, peso propio medio del forjado, en $\mathbf{k N} / \mathbf{m}^{\mathbf{2}}$.

\section{SOLADO MÁS REVESTIMIENTO DE TECHOS}

Tanto el solado como el revestimiento de techos son también variables probabilistas cuyos parámetros estadísticos, pesos medios, desviaciones estándar y coeficientes de variación, pueden ser obtenidos, al igual que el peso propio del forjado, mediante combinación de otras variables, producto de espesores por pesos específicos en cada una de las capas de los elementos constructivos multicapa, y suma de las variables que serían los pesos de cada una de las capas.

Aplicando los mismos criterios de los códigos probabilistas y la Norma EN-1991-1-1 (1), adoptaremos, al igual que para el peso propio, como valores representativos de las acciones de peso propio del solado y del revestimiento de techos, los pesos de los elementos constructivos con sus valores medios, es decir los deducidos de los volúmenes calculados con sus dimensiones nominales y los pesos específicos medios de los materiales empleados. Para las dimensiones representativas o nominales adoptaremos sus valores medios. Estos valores nominales serán los definidos en el proyecto y entendemos que los parámetros peso específico y su coeficiente de variación o su desviación estándar deberían ser objeto de especificación en los códigos de acciones probabilistas, al igual que señalamos para el peso propio del forjado.

Conocida pues la media de las acciones de peso propio de estas unidades constructivas, solado mas revestimiento de techos, al igual que señalamos para el peso propio de la estructura, para tener caracterizada la variable precisamos conocer la desviación estándar, con lo cual también tendríamos conocido el coeficiente de variación, y el tipo de distribución al que se ajusta. 
También para estas acciones, de carácter permanente, la distribución que generalmente se sugiere aplicar es la distribución Normal y es la que adoptaremos.

Resultaría aplicable para el cálculo de las desviaciones estándar o coeficientes de variación, lo que expusimos para el peso propio de la estructura, diferenciando el proceso correspondiente a una situación de dimensionado o comprobación en fase de proyecto de la situación de comprobación de una estructura existente, ya que en el segundo caso podríamos obtener tanto el el valor del peso propio medio como de la desviación estándar de estas acciones realizando un muestreo significativo sobre la construcción existente y con los datos tomados de tal muestreo estimar los parámetros estadísticos de estas variables.

Si nos centramos en el caso de proyecto o de comprobación de la estructura en caso de proyecto al igual que para el peso propio necesitamos:

- Conocer las dimensiones nominales para las que se adoptan los espesores medios de cada capa para calcular sus volúmenes medios $\left(\mathbf{V}_{\mathbf{M}}\right)$ por unidad de superficie.

- Conocer las desviaciones estándar y coeficientes de variación de estos espesores que son datos que, al igual que los de la estructura, deberían figurar en los códigos estructurales. Conocidas las desviaciones estándar y los coeficientes de variación de las dimensiones, como combinación de variables podríamos conocer las desviaciones estándar $\left(\boldsymbol{\sigma}_{\mathrm{v}}\right)$ de los volúmenes y sus coeficientes de variación $\left(\boldsymbol{\delta}_{\mathrm{v}}\right)$.

- Conocer el peso específico medio $\left(\mathbf{P}_{\mathbf{e s p}, \mathbf{M}}\right)$ del material de cada capa, su desviación estándar ( $\left.\boldsymbol{\sigma}_{\text {Pesp }}\right)$ y su coeficiente de variación, $\left(\boldsymbol{\delta}_{\text {Pesp }}\right)$, datos que asimismo deberían estar especificados en los códigos estructurales.

Conocidos los datos anteriores es posible conocer los valores medios $\left(\mathbf{p}_{\mathbf{M C}}\right)$ de los pesos de cada una de las capas que constituyen el solado y el revestimiento de techos, así como su desviación estándar $\left(\boldsymbol{\sigma}_{\mathrm{C}}\right)$ y su coeficiente de variación $\left(\boldsymbol{\delta}_{\mathrm{C}}\right)$ como variable estadística producto de las variables volumen y peso específico del material (ecuaciones [7], [8] y [9]):

$$
\begin{gathered}
\mathbf{p}_{\mathrm{MC}}=\mathbf{V}_{\mathrm{M}, \mathrm{C}} \cdot \mathbf{P}_{\mathrm{esp} \mathrm{C}, \mathrm{M}} \\
\boldsymbol{\delta}_{\mathrm{C}}=\left(\boldsymbol{\delta}_{\mathrm{V}}{ }^{2}+\boldsymbol{\delta}_{\mathrm{Pesp}}{ }^{2}\right)^{\mathbf{o} \cdot 5} \\
\boldsymbol{\sigma}_{\mathrm{C}}=\boldsymbol{\delta}_{\mathrm{C}} \cdot \mathbf{p}_{\mathrm{MC}}
\end{gathered}
$$

Componiendo mediante la suma de variables de cada capa los parámetros tendríamos la variable aleatoria correspondiente a la carga permanente de pesos del solado mas el revestimiento de techos, haciendo la observación que el análisis también podría ser realizado mediante paquetes de capas (ecuaciones [10], [11] y [12]):

$$
\begin{gathered}
\mathbf{p}_{\mathrm{M}, \text { solado+rev }}=\Sigma \mathbf{P}_{\mathrm{M}, \mathrm{C}} \\
\sigma_{\text {solado+rev }}=\left(\Sigma \sigma_{\mathrm{C}}^{2}\right)^{\mathbf{o . 5}} \\
\boldsymbol{\delta}_{\text {solado }}=\sigma_{\text {solado+rev }} / \mathbf{p}_{\mathrm{M}, \text { solado+rev }}
\end{gathered}
$$

Hemos desarrollado un análisis paramétrico para un tipo de solado tradicional, empleado en edificación en España, formado por una capa de arena, capa de mortero y solado de terrazo, con unos espesores nominales usuales y especificados en fase de proyecto como valores medios. El revestimiento de techo, unidad normalmente de menor incidencia en el cálculo estructural al ser su peso de valor mucho más reducido que el del solado y tener menor variabilidad, le asignamos el carácter de determinista, lo que nos facilita el análisis numérico de los parámetros que tenemos que calcular. En la Figura 5 acompañamos la tipología del solado que como ejemplo hemos adoptado y en la Tabla 4 la indicación de sus dimensiones nominales y la variabilidad de los espesores de sus capas que hemos considerado. Contemplando el solado mas el revestimiento de techos como un paquete y adoptando como valores deterministas en este caso los pesos específicos de los materiales que componen todas las capas, dado que la variabilidad que condiciona casi totalmente los valores de los parámetros son los espesores de las mismas, hemos calculado el peso propio medio, la desviación estándar y el coeficiente de variación de este tipo de solado mas el revestimiento de techos.

Tabla 4. Dimensiones nominales y variabilidade de los espesores

\begin{tabular}{|c|c|c|}
\hline $\begin{array}{c}\text { Cama de arena } \\
\mathbf{c m}\end{array}$ & $\begin{array}{c}\text { Capa de mortero } \\
\mathbf{c m}\end{array}$ & $\begin{array}{c}\text { Solado de terrazo } \\
\mathbf{c m}\end{array}$ \\
\hline $\mathrm{e}_{\mathrm{A}}-1,0$ & $\mathrm{e}_{\mathrm{M}}-1, \mathrm{O}$ & $\mathrm{e}_{\mathrm{T}}-0,5$ \\
\hline $\mathrm{e}_{\mathrm{A}}=3 \mathrm{~cm}$ & $\mathrm{e}_{\mathrm{M}}=2,0 \mathrm{~cm}$ & $\mathrm{e}_{\mathrm{T}}=2,0 \mathrm{~cm}$ \\
\hline $\mathrm{e}_{\mathrm{A}}+1, \mathrm{O}$ & $\mathrm{e}_{\mathrm{M}}+1,0$ & $\mathrm{e}_{\mathrm{T}}+0,5$ \\
\hline 3 casos & 3 casos & 3 casos \\
\hline \multicolumn{3}{|c}{ Total 27 casos } \\
\hline
\end{tabular}

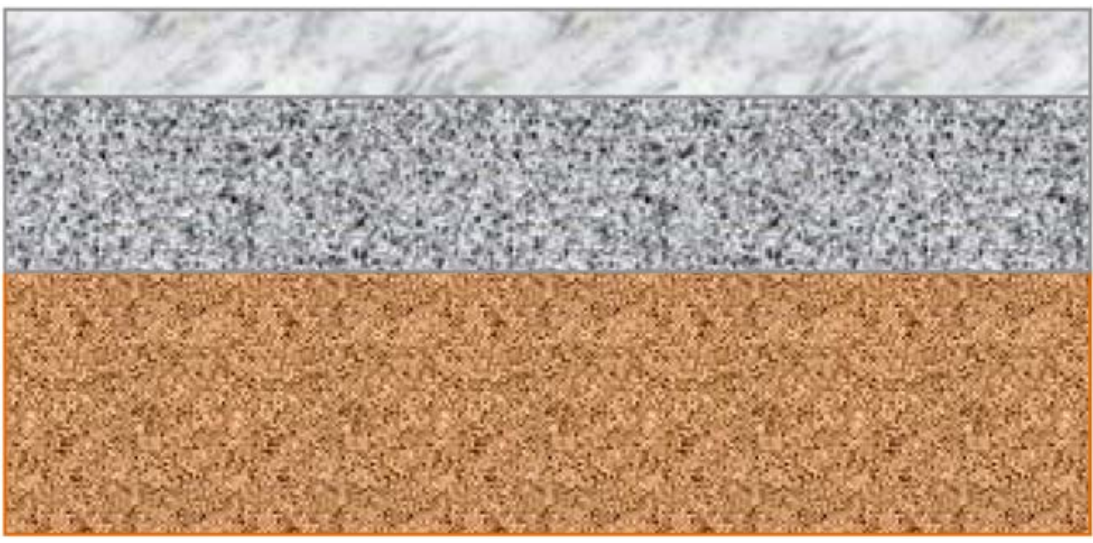

Solado

Figura 5. Tipología del solado. 
En la Tabla 5 resumimos los valores obtenidos del análisis paramétrico del que se deducen los valores de los parámetros necesarios para caracterizar la acción de peso propio del solado más revestimiento de techos, valor medio de la acción, $\mathbf{p}_{\mathrm{M}, \mathbf{S}+\mathbf{R}}=\mathbf{1 , 5 0 0} \mathbf{k N} / \mathbf{m}^{2}$, coeficiente de variación, $\boldsymbol{\delta}_{\mathrm{S}+\mathrm{R}}=\mathbf{0 , 1 5 5}$ y desviación estándar, $\boldsymbol{\sigma}_{\mathrm{S}+\mathrm{R}}=\mathbf{0 , 2 3 3} \mathbf{k N} / \mathbf{m}^{2}$, proponiendo adoptar los valores siguientes para este tipo de solado y revestimiento de techo (ecuaciones [13], [14] y [15]):

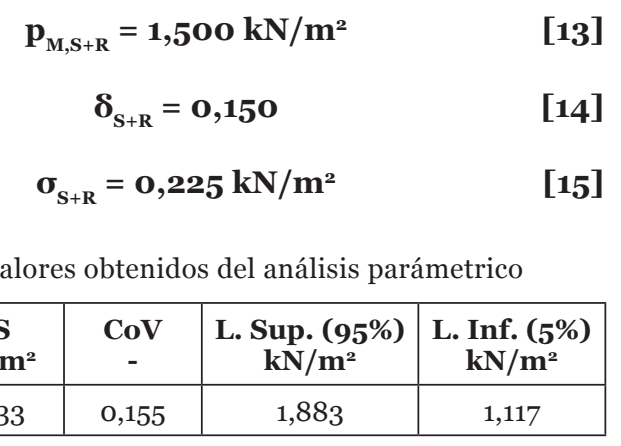

La metodología expuesta en este ejemplo puede ser aplicada a otros tipos de solado. Asimismo, podríamos haber realizado el ejemplo mediante suma de capas y no contemplando el paquete de capas en su conjunto, aunque estimamos que el análisis realizado es suficientemente aproximado y los valores de los parámetros $\boldsymbol{\delta}_{\mathbf{S}+\mathbf{R}}$ y $\boldsymbol{\sigma}_{\mathbf{S}+\mathbf{R}}$ no se desviarían de los calculados de forma significativa.

La metodología expuesta podría ser aplicada para que los códigos de acciones incorporasen los valores de los pesos medios y coeficientes de variación de diferentes tipos de solado nominales.

Como se ve los solados, aunque el valor de las acciones medias que suponen sean mas reducidas que los pesos propios de los forjados, presentan en general unos coeficientes de variación muy superiores, lo que conduce a que en un análisis estadístico de acciones máxima y mínima probables, representado por fractiles del $95 \%$ y del $5 \%$ de verse superadas, se obtengan los resultados característicos $\mathbf{p}_{\mathrm{KN}, \mathbf{0 . 9 5}, \mathrm{S}+\mathrm{R}}=\mathbf{1 , 1 1 7} \mathbf{~ k N} / \mathbf{m}^{2}$ y $\mathbf{p}_{\mathrm{KN}, \mathbf{0 . 0}, \mathrm{S}+\mathbf{R}}=\mathbf{1}, \mathbf{8 8 3} \mathbf{k N} / \mathbf{m}^{2}$ según se señala en la Tabla 4 muy alejados obviamente de los valores medios.

Por último, queremos llamar la atención sobre la importancia que tendría la correcta evaluación de estos parámetros que caracterizan al solado en los análisis de construcciones existentes. En estos casos las desviaciones sobre los valores medios, es decir su variabilidad, pueden ser mucho mas significativas aún, debidas a causas como por ejemplo la superposición de pavimentos y la corrección de deformaciones estructurales por fluencia a base de recrecidos de las capas del solado significativos. Estos aspectos en algunos casos pueden condicionar fuertemente la seguridad estructural y por ello el proceso de selección de la muestra debe tomarlos en consideración para que tanto los valores medios como su coeficiente de variación incorporen sus efectos, seleccionando los puntos de muestreo de forma que la muestra sea significativa.

\section{TABIQUERÍA}

Se entiende como acción de tabiquería la carga de carácter permanente originada por las fábricas de distribución y sus revestimientos en obras de edificación. Estas acciones no tendrían el carácter pleno de permanentes, ya que en rea- lidad serían variables en el tiempo y en el espacio, dada la posibilidad, aceptada por los códigos de acciones, de que la distribución sea modificable a lo largo de la vida útil de la edificación. No se trataría además de una acción uniformemente distribuida actuante sobre los forjados, sino que se trata de un conjunto de cargas lineales transmitidas a su soporte por elementos de gran rigidez al ser asimilables los tabiques a vigas de gran canto.

Los aspectos expuestos hacen que el peso de la tabiquería sea una acción de carácter muy complejo, para cuya consideración y caracterización las normas de acciones plantean simplificaciones con objeto de hacer viable y facilitar el análisis estructural.

Los códigos de acciones antiguos fijaban normalmente como acción nominal de tabiquería el valor de $1,00 \mathrm{kN} / \mathrm{m}^{2}$ aplicada a la superficie del forjado cuando la sobrecarga de uso de la edificación era de $2,00 \mathrm{kN} / \mathrm{m}^{2} \mathrm{o}$ inferior. Este valor se reducía a $0,50 \mathrm{kN} / \mathrm{m}^{2}$ cuando la sobrecarga de uso de era de $3,00 \mathrm{kN} /$ $\mathrm{m}^{2}$, y permitían no considerarla cuando la sobrecarga de uso era igual o superior a $4,00 \mathrm{kN} / \mathrm{m}^{2}$.

Los códigos de acciones más actuales y en particular el Código Técnico de la Edificación, CTE (2), establece un nuevo criterio especificando textualmente: "Para el caso de tabiques ordinarios cuyo peso por $\mathrm{m}^{2}$ no sea superior a $1,20 \mathrm{kN} /$ $\mathrm{m}^{2}$, su grueso o espesor no exceda de $8 \mathrm{~cm}$ y su distribución en planta sea sensiblemente homogénea, su peso propio podrá asimilarse a una carga equivalente uniformemente distribuida. Como valor de dicha carga equivalente se podrá adoptar el valor $0,8 \mathrm{kN} / \mathrm{m}^{2}$ multiplicado por la razón media entre la superficie de tabiquería y la de la planta considerada. En el caso de tabiquería más pesada, esta podrá asimilarse al mismo valor de la carga equivalente uniforme citado más un incremento local, de valor igual al exceso de peso del tabique respecto a $1,00 \mathrm{kN} / \mathrm{m}^{2}$ de alzado" añadiendo en otro apartado que "en general en viviendas bastará considerar como peso propio de la tabiquería una carga de 1, oo $\mathrm{kN} / \mathrm{m}^{2}$ de superficie construida".

Por su parte el International Building Code (IBC) (2017 Edition) (3) en su apartado 1607.5 Partition Loads establece una acción equivalente uniformemente distribuida debida a la tabiquería de $15 \mathrm{psf}\left(0,72 \mathrm{kN} / \mathrm{m}^{2}\right)$ para los casos en que la sobrecarga de uso sea inferior a $80 \mathrm{psf}\left(3,83 \mathrm{kN} / \mathrm{m}^{2}\right)$, incluyéndola en el apartado de sobrecargas si se trata de tabiquerías que pueden ser modificadas (Live Load).

La Norma sobre Acciones en Estructuras EN 1991-1-1 (1) en su apartado 6.3.1.2 (8) establece una acción de tabiquería por $\mathrm{m}^{2}$ de forjado construido, suponiendo que el forjado dispone de capacidad de reparto transversal de la carga lineal actuante, que es función del peso propio por ml lineal que supone la tabiquería; $0,5 \mathrm{kN} / \mathrm{m}^{2}$ para cargas por $\mathrm{ml}$ de tabique menor de $1,00 \mathrm{kN} / \mathrm{m} ; 0,8 \mathrm{kN} / \mathrm{m}^{2}$ para peso de tabique entre $1 \mathrm{y}$ $2 \mathrm{kN} / \mathrm{m}$ y $1,20 \mathrm{kN} / \mathrm{m}^{2}$ para peso de tabique entre 2 y $3 \mathrm{kN} / \mathrm{m}$.

Se aprecian pues diferencias entre los códigos de acciones a que hemos hecho referencia: en primer lugar, la asignación del carácter de la carga de tabiquería, contemplada como acción de carácter permanente en el CTE, como sobrecarga en EN 1991-1-1 y como carga no permanente en el IBC (Live Load), y en segundo lugar el propio valor de la acción, inferior en IBC que en el CTE y en EN 1991-1-1. Hay otro aspecto 
también importante y es que la regla simplificada establecida en el CTE de considerar una acción debida a la tabiquería de $1,00 \mathrm{kN} / \mathrm{m}^{2}$ de superficie construida, es aplicable al uso como viviendas pero no se cita que sea aplicable a otros usos, como oficinas con sobrecarga de uso $3,00 \mathrm{kN} / \mathrm{m}^{2}$, en las cuales la densidad de tabiquería suele ser sensiblemente inferior.

En el contexto en que estamos analizando esta acción, que debe ser especificada de cara a un análisis probabilista y dado la complejidad que conceptualmente puede entrañar su especificación, estableceremos los siguientes criterios para su caracterización y para establecer los valores de sus parámetros:

- Fijaremos como valor nominal y característico de la acción el valor medio del peso $/ \mathrm{m}^{2}$ de la tabiquería, el cual será considerado como determinista.

- La variabilidad de la acción será debida, fundamentalmente, a la densidad de $\mathrm{m}^{2}$ de tabique existente por $\mathrm{m}^{2}$ de forjado construido, lo cual a su vez será función del tamaño de las dependencias de la edificación que delimitan los tabiques.

- Los valores medios de la carga uniforme de tabiquería se calcularán por dependencias, evaluando la carga media en un forjado por unidad de vivienda.

- En cada vivienda se calcularía el coeficiente de variación en función de los tamaños de las dependencias.

Hemos desarrollado un análisis paramétrico de diferentes tipos de viviendas, con diferentes dependencias separadas por tabiques cuyo peso por $\mathrm{m}^{2}$ de alzado sea una variable determinista. Para cada dependencia se calcula el índice de tabiquería deducido de dividir los $\mathrm{m}^{2}$ de tabique que se asignan a cada dependencia por los $\mathrm{m}^{2}$ en planta de la misma. Estos índices tendrán unos valores variables cuya variabilidad condiciona la variabilidad de la acción.
La acción de tabiquería se ha considerado como el valor medio de la repercusión de la tabiquería en $\operatorname{los}^{2}$ de la vivienda y como coeficiente de variación de la acción se adoptará el correspondiente al índice de tabiquería, contemplando en el análisis unos determinados tipos de vivienda con superficies variables.

En la Figura 6 acompañamos un esquema de la dependencia tipo sobre la cual hemos calculado el que hemos denominado como índice de tabiquería, en función de las dimensiones variables de la dependencia.

En la Figura 7 acompañamos un resumen de los resultados del análisis paramétrico realizado, de los que puede deducirse la escasa variabilidad del coeficiente de variación para viviendas de superficie superior $90 \mathrm{~m}^{2}$ o 6 dependencias, para las cuales se puede estimar un coeficiente de variación de la tabiquería con valor 0,20, pudiendo llegar hasta 0,25 para superficies de vivienda reducidos, inferiores a $60 \mathrm{~m}^{2}$.

Por otra parte la repercusión media de la tabiquería, valorada por el índice de tabiquería antes definido, se sitúa en órdenes de 1,10. Este valor supone una acción media debida a la tabiquería de $0,88 \mathrm{kN} / \mathrm{m}^{2}$, para un peso del tabique de $0,8 \mathrm{kN} / \mathrm{m}^{2}$, valor intermedio entre los que son especificados por el CTE, $\left(1,00 \mathrm{kN} / \mathrm{m}^{2}\right)$ y el IBC $\left(0,72 \mathrm{kN} / \mathrm{m}^{2}\right)$ si a tales valores nominales les asignamos el carácter de valores medios. Aplicando la EN 1991-1-1 la acción de tabiquería de $1.00 \mathrm{kN} /$ $\mathrm{m}^{2}$ correspondería a un caso intermedio entre cargas lineales medias transmitidas por el tabique de 1,00 y $1,20 \mathrm{kN} / \mathrm{m}$.

Con lo expuesto tenemos una base suficiente para poder establecer los valores de los parámetros que precisamos para caracterizar la variable acción originada por la tabiquería, es decir su valor medio, su coeficiente de variación y su desvia-

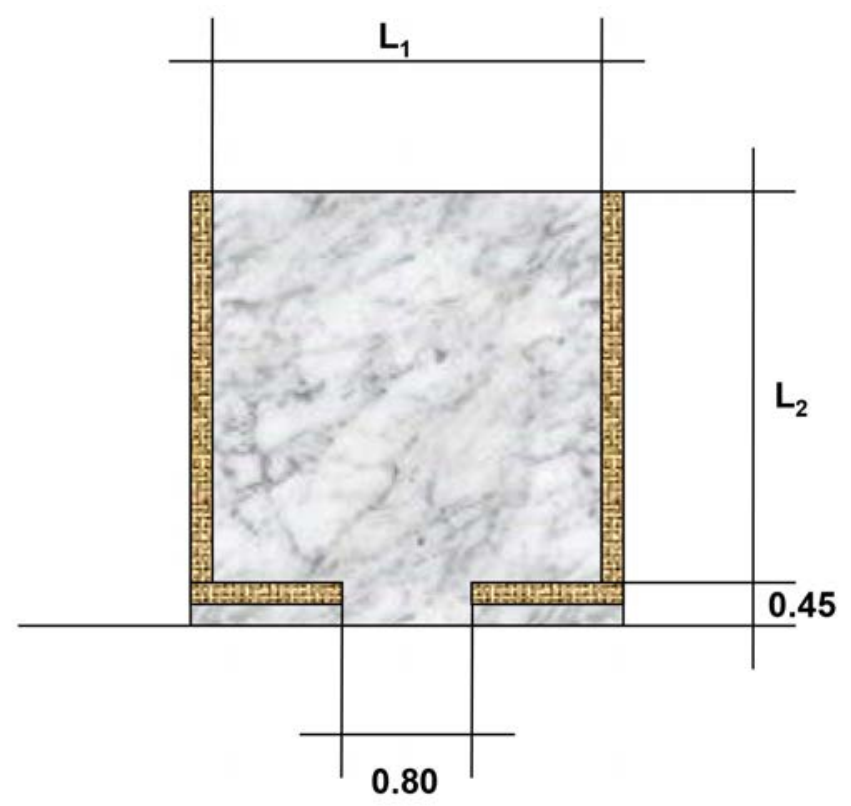

$\left(M^{2}\right.$ de Tabique $) /\left(M^{2}\right.$ de Forjado $)=\left(\left(0.5 \cdot 2 \cdot L_{2}+L_{1}-0,80\right) \cdot H\right) /\left(L_{1} \cdot\left(L_{2}+0.45\right)\right)$

\author{
$L_{1}$ y $L_{2}$ Variables
}

Figura 6. Esquema de la dependencia tipo. 


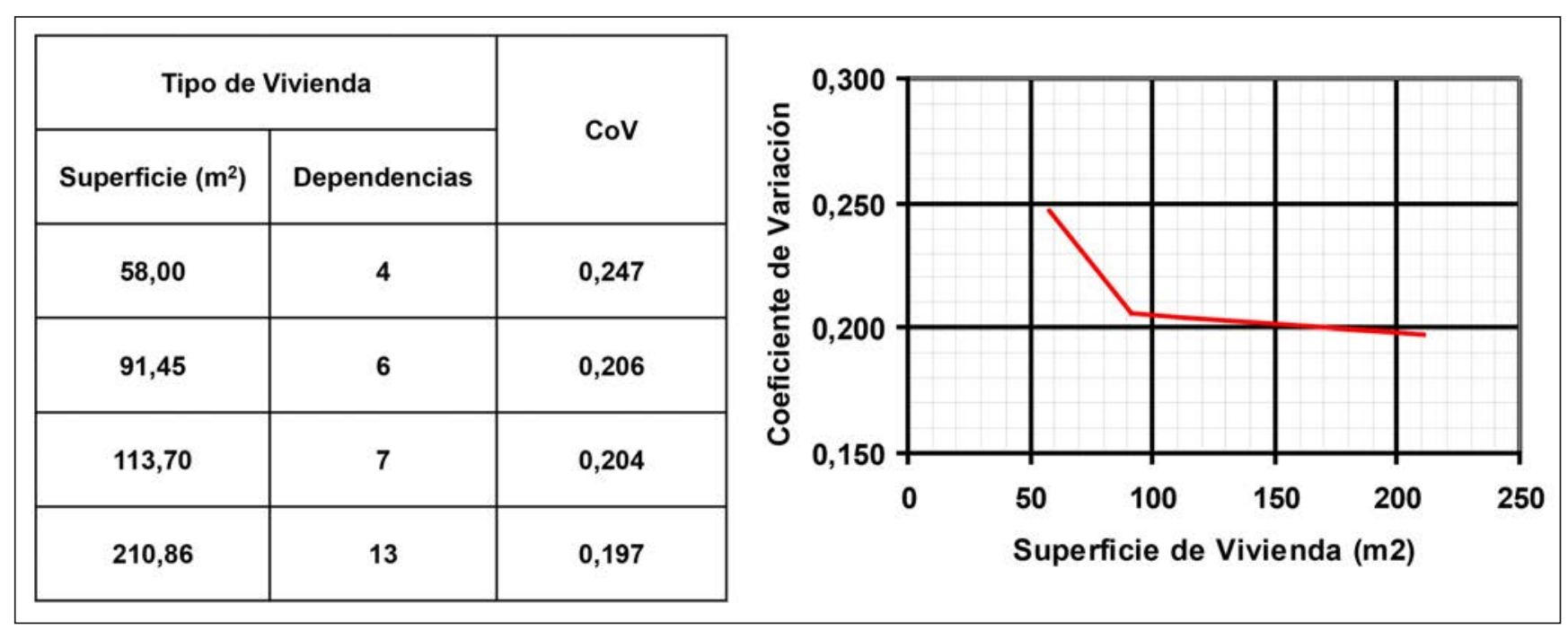

Figura 7. Resultados del análisis parámetrico.

ción estándar. Según ello para la variable acción de tabiquería en viviendas pueden adoptarse unos parámetros cuyos valores serían las de las ecuaciones [16], [17] y [18]:

$$
\begin{aligned}
& \text { Acción Media } p_{\mathrm{MT}}=1,00 \mathrm{kN} / \mathrm{m}^{2} \\
& \text { Coeficiente de Variación } \delta_{\mathrm{T}}=0,20 \\
& \text { Desviación Estandar } \sigma_{\mathrm{T}}=0,20 \mathrm{kN} / \mathrm{m}^{2}
\end{aligned}
$$

en viviendas de tamaño superior a $90 \mathrm{~m}^{2}$ de superficie y siempre que el peso de la tabiquería no exceda $0,80 \mathrm{kN} / \mathrm{m}^{2}$ de alzado, corrigiendo el valor medio si la tabiquería excede el peso señalado. En ese caso se incrementaría la acción media en el valor que exceda el peso del tabique sobre $1,00 \mathrm{kN} / \mathrm{m}^{2}$ según establece el CTE.

En el caso de edificaciones destinadas a oficinas o a otros usos, en los que sobrecarga de uso nominal sea de $3,00 \mathrm{kN} /$ $\mathrm{m}^{2}$, sugerimos adoptar un valor medio de la acción de tabiquería que sea el 50\% del valor propuesto, con la corrección por peso del tabique al que antes hicimos referencia según el CTE, y que para los casos de usos cuya sobrecarga de utilización supere $4,00 \mathrm{kN} / \mathrm{m}^{2}$ se prescinda de la sobrecarga de tabiquería en el análisis de la solicitación.

\section{CONCLUSIONES}

De acuerdo con el análisis preliminar realizado establecemos las conclusiones siguientes:

- Las acciones especificadas en los códigos estructurales para el peso propio del forjado y vigas de la estructura, solado mas revestimiento de techos y tabiquerías, en estructuras de edificación, tienen un "carácter de acciones medias, las cuales pueden ser evaluadas a partir de las dimensiones nominales medias y los pesos específicos medios de los materiales que las integran" según se establece en las normas de acciones

- Para el caso del peso propio de los forjados unidireccionales nervados, con losa superior de espesor $5 \mathrm{~cm}$, nervios de hormigón y aligeramientos cerámicos, hemos aproximado los valores del peso propio medio $\left(\mathbf{p p}_{\mathrm{M}}\right)$ y de su coeficiente de variación $\left(\boldsymbol{\delta}_{\mathrm{pp}}\right)$ a partir de un análisis paramétrico va- riando las dimensiones básicas estructurales y adoptando unos pesos de carácter determinista para los aligeramientos. Este análisis conduce a las formulaciones siguientes (ecuaciones [19] y [20]):

$$
\begin{gathered}
\mathbf{p p}_{\mathrm{M}, \text { forjado }}=1,40+0,06 \cdot \mathrm{h} \\
\delta_{\text {forjado }}=0,9 \cdot(0,1-0,001 \cdot h)
\end{gathered}
$$

siendo $\mathbf{h}$ el canto del forjado en $\mathrm{cm}$, para $\mathrm{pp}_{\mathrm{M}}$ en $\mathrm{kN} / \mathrm{m}^{2}$.

- Para una acción debida a los revestimientos de techos a base de placas de yeso de $1 \mathrm{~cm}$ de espesor hemos evaluado, mediante la realización de análisis paramétrico, los valores medios y los coeficientes de variación de los pesos de los solados mas los revestimientos de techos tradicionalmente mas empleados en edificación, constituidos los solados a base una cama de arena de $3 \mathrm{~cm}$, capa de mortero de $2 \mathrm{~cm}$ y pavimento de losetas de terrazo de espesor entre 1,5 y 2,5 cm. A partir de los resultados obtenidos proponemos considerar para estos parámetros estadísticos los siguientes valores (ecuaciones [21] y [22]):

$$
\begin{gathered}
\mathrm{pp}_{\mathrm{M}, \text { solado }}=1,50 \mathrm{kN} / \mathrm{m}^{2} \\
\boldsymbol{\delta}_{\text {soladp }}=0,15
\end{gathered}
$$

- Por último, para la tabiquería hemos evaluado, mediante la realización de un análisis paramétrico variando los $\mathrm{m}^{2}$, la distribución y el número de dependencias de las viviendas, el valor del coeficiente de variación, proponiendo los valores siguientes para estos parámetros estadísticos (ecuaciones [23] y [24]):

$$
\begin{aligned}
\mathrm{pp}_{\mathrm{M}, \text { tabiqueria }} & =\mathrm{p} \mathrm{kN} / \mathrm{m}^{2} \\
\delta_{\text {tabiqueria }} & =0,20
\end{aligned}
$$

siendo $\mathbf{p}_{\mathbf{M} \text {,tabiqueria }}=\mathbf{1}, \mathbf{0 0} \mathbf{k N} / \mathbf{m}^{\mathbf{2}}$ para viviendas, valor que deberá ser corregido si el propio de la tabiquería por $\mathrm{m}^{2} \mathrm{de}$ alzado supera los $0,8 \mathrm{kN} / \mathrm{m}^{2}$ de acuerdo con lo indicado en el CTE, incrementado la acción media debida a la tabiquería en la misma proporción que se incrementa su peso frente al valor nominal medio de $0,8 \mathrm{kN} / \mathrm{m}^{2}$ considerado. Recomendamos que para viviendas con superficie infe- 
rior a $75 \mathrm{~m}^{2}$ se adopte un valor del coeficiente de variación $\boldsymbol{\delta}_{\text {tabiqueria }}=\mathbf{0 , 2 5}$.

- El procedimiento que se propone podría ser aplicado para caracterizar de una manera simple las acciones de peso propio y carga permanente como variables estadísticas para realizar los análisis de fiabilidad estructural sobre estructuras de edificios ya construidos tomando datos directos de la construcción.

\section{REFERENCIAS}

(1) CEN (2002): Eurocódigo 1 (EC-1): EN 1991-1-1. Actions on structures-Part 1-1: General actions-Densities, self-weight, imposed loads for buildings. Comité Europeo de Normalización.

(2) Ministerio de Vivienda (2006): Código Técnico de la Edificación (CTE). Ministerio de Fomento. Madrid. 2006.

(3) International Code Council (ICC) (2017): International Building Code (IBC). ICC. Illinois. USA.

(4) Ministerio de Fomento (2008): Instrucción Española de Hormigón Estructural (EHE-o8). Secretaria General Técnica del Ministerio de Fomento. Madrid. 2008.

(5) CEN (2004): Eurocódigo 2 (EC-2): EN 1992-1-1. Design of concrete structures-Part 1-1: General rules and rules for buildings. Comité Europeo de Normalización.

(6) FIB (2013): Model Code for concrete structures 2010. Federation International du Beton. Lausanne. Ernst\&Sohn.

(7) ACI (2014): ACI 318-14 Building code requirements for structural concrete. American Concrete Institute. USA. 